\title{
NONHOMOGENEOUS POLYADIC ALGEBRAS
}

\section{LEON LEBLANC}

Introduction. In a series of papers [1-4] Halmos introduced the theory of homogeneous polyadic algebras. These polyadic algebras correspond to first order calculi in which there is only one sort of variables. The purpose of this paper is to introduce the theory of nonhomogeneous polyadic algebras, the latter algebras corresponding to first order calculi in which there are several sorts of variables; these calculi were first treated in $[6 ; 7]$. In $\S 1$, we develop the general theory; in $\$ 2$, we offer a representation theorem which proof, even in the homogeneous case, is of some novelty; finally, in $\$ 3$, we show that, to a certain extent, the theory of finite-sorted nonhomogeneous algebras can be reduced to that of homogeneous algebras.

0 . Notation. The purpose of this section is to set down the notation to be used throughout. However, we shall restrict attention to the notation which is of some novelty and which is more or less proper to this paper. For the standard notation concerning Boolean algebras and homogeneous polyadic algebras, we refer to the four papers of Halmos [1-4]. Let $I$ be a nonempty set. For our purpose, it will be convenient to view a partition of $I$ as a mapping $U$ from a set $M$ to subsets $U_{\alpha}$ of $I$ such that the union of the sets $U_{\alpha}$, as $\alpha$ runs through $M$, is $I$ and such that $U_{\alpha} \cap U_{\beta}=\varnothing$ whenever $\alpha \neq \beta$; the set $M$ shall be called the domain of $U$. For the remainder of this section, the symbols $I, U$ and $M$ shall retain the meaning they have just been assigned. If $X$ is a mapping from $I$ to sets such that $X_{i}=X_{j}$ whenever $i$ and $j$ belong to the same set $U_{\alpha}$, then we shall denote by $X_{I}$ the cartesian product of the sets $X_{i}$ as $i$ runs through $I$. If $i$ and $j$ are elements in $I$, then the symbol $(j / i)$ shall denote the transformation that sends $i$ on to $j$ and all other elements onto themselves. Previously, in [2-4], this transformation was what is denoted now by $(i / j)$. The advantage of this new notation is that the "cancellation law" holds: $S(j / k) S(k / i) p=S(j / i) p$ whenever $p$ belongs to some polyadic algebra and $i, j$ and $k$ are variables so that $p$ is independent of $k$. A transformation $\tau$ on $I$ shall be called a $U$-transformation if $\tau\left(U_{\alpha}\right)$ $\subseteq U_{\alpha}$ whenever $\alpha \in M$. Let $\tau$ be a $U$-transformation on $I ; \tau_{*}$ shall denote the mapping of $X_{I}$ into $X_{I}$ induced by $\tau$. By definition, $\left(\tau_{*} x\right)_{i}$ $=x_{\tau i}$ for all $x$ in $X_{I}$ and all $i$ in $I$. If $x$ and $y$ are in $X_{I}$ and if $J$ is a subset of $I$, then $x \equiv y$ mod $J$ shall mean that $x_{i}=y_{i}$ for $i$ not in $J$.

Received by the editors, January 30, 1961. 
The letter $O$ shall be used to denote the two-element Boolean algebra. On one occasion we shall use the McNeille completion of a Boolean algebra; more precisely, if $B$ is a Boolean algebra, then there exists a unique complete Boolean algebra $A$ so that $B$ is a subalgebra of $A$ and every element in $A$ is the supremum of some subset of $B$. We shall refer to $A$ as the McNeille completion of $B$. For the details, see [5].

1. Elementary theory. In this section, we introduce the basic concepts of the theory of nonhomogeneous algebras. We start by giving the definition of a nonhomogeneous polyadic algebra. If $M$ is a nonempty set, then an $M$-sorted nonhomogeneous polyadic algebra is a quintuple $(A, I, U, S, \exists$ ) where $A$ is a Boolean algebra, $I$ is a set, $U$ is a partition of $I$ with domain $M, S$ is a mapping from $U$-transformations to endomorphisms of $A$, and $\exists$ is a mapping from subsets of $I$ to quantifiers of $A$ such that

$\left(\mathrm{P}_{1}\right)$ if $\delta$ is the identity transformation on $I$, then $S(\delta)$ is the identity endomorphism, $I$,

$\left(\mathrm{P}_{2}\right) S(\tau \sigma)=S(\tau) S(\sigma)$ whenever $\tau$ and $\sigma$ are $U$-transformations on

$\left(\mathrm{P}_{8}\right)$ if $\varnothing$ is the empty subset of $I$, then $\exists(\varnothing)$ is the discrete quantifier,

$\left(\mathrm{P}_{4}\right) \exists(J \cup K)=\exists(J) \exists(K)$ whenever $J$ and $K$ are subsets of $I$,

$\left(\mathrm{P}_{\mathrm{b}}\right)$ if $\tau$ and $\sigma$ are $U$-transformations on $I$, and if $\tau=\sigma$ outside $J$ where $J$ is a subset of $I$, then $S(\tau) \exists(J)=S(\sigma) \exists(J)$,

$\left(\mathrm{P}_{6}\right)$ if $\tau$ is a $U$-transformation on $I$, if $J$ is a subset of $I$ and $\tau$ is one-to-one on $\tau^{-1}(J)$, then $\exists(J) S(\tau)=S(\tau) \exists\left(\tau^{-1} J\right)$.

The algebra $(A, I, U, S, \exists)$ shall be referred to as an $(I, U)$ algebra when the explicit mention of $M$ and of the operator mappings $S$ and $\exists$ does not seem necessary. As in the homogeneous case, we shall of ten commit the solecism of identifying $(A, I, U, S, \exists)$ with $A$ itself. Elements of $M$ will be called sorts and they shall be denoted by small Greek letters. Elements of $I$ will be called variables and the notation for variables, subsets of variables, and the operator mappings shall be the same as in the homogeneous case. A variable will be called of the sort $\alpha$ if it belongs to the set $U_{\alpha}$. Observe that if $M$ is a singleton, then $A$ is an ordinary homogeneous $I$-algebra. It is also clear that any homogeneous $I$-algebra becomes, in a natural way, a nonhomogeneous algebra after declaring all variables of $I$ to be of the same sort. The $\alpha$-degree of $A$ is the cardinality of the set $U_{\alpha}$; the degree of $A$ is the smallest $\alpha$-degree of $A$ as $\alpha$ runs over $M$. The concepts of independence, support and local-finiteness are defined as in the homogeneous case. The elementary algebraic theory for non- 
homogeneous algebras (i.e., definitions of subalgebras, ideals, quotient algebras, etc.) is entirely similar, up to trivial and obvious modifications, to the corresponding theory for homogeneous algebras (see $[2, \S 8])$. Consequently, we shall not develop it here and we shall make free use of it in the sequel. One of the more efficient ways to construct nontrivial examples of nonhomogeneous algebras is to refer to functional algebras. The notion of functional algebra is also needed to formulate and prove the representation theorem for nonhomogeneous polyadic algebras. Let $I$ be a set, $U$ a partition of $I$ with domain $M, X$ a mapping from $I$ to sets $X_{i}$ such that $X_{i}=X_{j}$ whenever $i$ and $j$ belong to the same set $U_{\alpha}$, and $B$ a Boolean algebra. If $p$ is a function from $X_{I}$ to $B$ and if $\tau$ is a $U$-transformation on $I$, define a function $S(\tau) p$ from $X_{I}$ to $B$ by $S(\tau) p(x)=p\left(\tau_{*} x\right)$ whenever $x$ belongs to $X_{I}$. Then $S(\tau)$ is a Boolean endomorphism (under pointwise operations) of the Boolean algebra of all functions from $X_{I}$ to $B$. Moreover, if $A$ is a Boolean algebra of functions from $X_{I}$ to $B$ and if $S(\tau)$ sends $A$ into $A$, then $S(\tau)$ is a Boolean endomorphism of $A$. To each subset $J$ of $I$ and each function $p$ from $X_{I}$ to $B$, we associate a function $\exists(J) p$ from $X_{I}$ to $B$ by $\exists(J) p(x)=\vee\{p(y): x \equiv y \bmod J\}$ whenever this last supremum exists for all $x$ in $X_{I}$. If $A$ is a Boolean algebra of functions from $X_{I}$ to $B$ and if $\exists(J) p$ is defined and belongs to $A$ for all $p$ in $A$, then $\exists(J)$ is a quantifier of $A$. A Boolean algebra $A$ of functions from $X_{I}$ to $B$ is a functional $M$-sorted nonhomogeneous (polyadic) algebra if $S(\tau) p$ belongs to $A$ and $\exists(J) p$ is defined and belongs to $A$ whenever $\tau$ is a $U$-transformation on $I, J$ is a subset of $I$ and $p$ belongs to $A$. We shall say then that $A$ is an $M$-sorted $B$-valued $(I, U)$-algebra over $X$. We shall also use expressions such as " $A$ is a functional nonhomogeneous algebra" or " $A$ is a functional $(I, U)$ algebra" etc. We shall indicate now how the concept of constant (see $[2, \S 12])$ is defined in nonhomogeneous algebras. Let $A$ be an $M$ sorted $(I, U)$-algebra and let $\alpha$ be a sort. An $\alpha$-constant of $A$ is a mapping $c$ from subsets $J$ of $U_{\alpha}$ to endomorphisms $S(c / J)$ of $A$ so that

( $\left.c_{1}\right) S(c / \phi)$ is the identity endomorphism,

(c) $S(c / J \cup K)=S(c / J) S(c / K)$,

(c) $S(c / J) \exists(H)=\exists(H) S(c / J-H)$,

(c) $\exists(H) S(c / J)=S(c / J) \exists(H-J)$,

(c) $S(c / J) S(\tau)=S(\tau) S\left(c / \tau^{-1} J\right)$,

whenever $J, K$ are subsets of $U_{\alpha}, H$ is a subset of $I$ and $\tau$ is a $U$ transformation on $I$. We shall also say that $c$ is a constant of the sort $\alpha$. The concept of richness is formulated (with the obvious modifications) exactly as in the homogeneous case. 
2. Representation. The proof of the representation theorem for (locally finite) homogeneous algebras (of infinite degree) given in [2] can easily be adapted to a proof of the corresponding fact for nonhomogeneous algebras. There is no point in repeating the details of that particular proof here. The purpose of this section is to offer a proof which, even in the homogeneous case, is of some novelty and is shorter than the original one. The proof is based on the following two lemmas.

(2.1) LemmA. If $A$ is an $M$-sorted locally finite $(I, U)$-algebra of infinite degree, $p$ an element of $A$ and $i$ a variable of the sort $\alpha$, then $\exists(i) p=\bigvee\left\{S(j / i) p: j \in U_{\alpha}\right\}$.

Proof. Fix the variables of $I-U_{\alpha}$ and apply $[2,10.5]$ to the (homogeneous) $U_{\alpha}$-algebra so obtained.

(2.2) Lemma. Suppose $A$ is an $M$-sorted $B$-valued $(I, U)$-algebra over $X$. If $B$ is complete and if $A$ is the algebra of all finite-dimensional functions from $X_{I}$ into $B$, then $A$ is rich.

Proof. It suffices to show that if $p$ has support $i$, then there exists a constant $c$ of the same sort as $i$ so that $\exists(i) p=S(c / i) p$. Suppose $p$ has support $i$ where $i$ is of the sort $\alpha$. Let $X_{i}=Z$ and let $\bar{p}$ be the natural function from $Z$ into $B$ induced by $p$. Well order the set $Z$ and let $e$ be the first element of $Z$ under that well-ordering. Define a mapping $\overline{\boldsymbol{r}}$ from $Z$ into $B$ by $\overline{\boldsymbol{r}}(e)=\bar{p}(e)$, and $\overline{\boldsymbol{r}}(a)=\bar{p}(a)-\mathrm{V}\{\overline{\boldsymbol{r}}(b): b<a\}$ if $a \neq e$. Define another mapping $\bar{q}$ from $Z$ into $B$ by $\bar{q}(e)=\bar{r}(e) \vee p_{0}$ where $p_{0}$ is the complement of $\bigvee\{\bar{p}(a): a \in Z\}$, and $\bar{q}(a)=\bar{r}(a)$ whenever $a \neq e$. Let $q$ be the unique element of $A$ with support $i$ and so that $q(x)=\bar{q}\left(x_{i}\right)$ for all $x$ in $X_{I}$. Define a mapping $f$ from $A$ into $A$ by $f p_{1}=\exists(i)\left(p_{1} \wedge q\right)$ for all elements $p_{1}$ in $A$. It is a straightforward matter to check that $f$ is actually a Boolean endomorphism of $A$ and that there exists a unique $\alpha$-constant $c$ of $A$ so that $S(c / i)=f$; moreover, $\exists(i) p=S(c / i) p$. This completes the proof of the lemma.

(2.3) THEOREM. If $A$ is a locally finite nonhomogeneous algebra of infinite degree, then $A$ is isomorphic to a functional algebra; if $A$ is simple, then $A$ is isomorphic to a O-valued functional algebra.

Proof. To prove the first part, suppose $A$ is an $M$-sorted $(I, U)$ algebra and define a mapping $X$ from $I$ into subsets of $I$ by $X_{i}=U_{\alpha}$ whenever $i$ is of the sort $\alpha$. Let $\tilde{A}$ be the Boolean algebra of all finitedimensional functions from $X_{I}$ into $A$ and define a mapping $f$ from $A$ into $\tilde{A}$ by $(f p)(\tau)=S(\tau) p$ for all $\tau$ in $X_{I}$ (note that a $U$-transformation on $I$ is an element of $X_{I}$ and conversely). Using (2.1), it is easy 
to check that $f(A)$ is a functional nonhomogeneous polyadic algebra and that $f$ is an isomorphism from $A$ onto $f(A)$. The latter proof is an adaptation to the nonhomogeneous case of $[2,10.9]$. To prove the second part, assume $A$ is simple. It suffices to prove that $A$ can be embedded in a rich algebra. This follows from the fact that the quotient of a rich algebra is rich and that a simple rich algebra is easily seen to be isomorphic to an 0 -valued functional algebra. To prove that $A$ can be embedded in a rich algebra, we may assume that $A$ is a $B$-valued functional algebra over $X$; this follows from the first part of the theorem. Let $\bar{B}$ be the McNeille completion of $B$ and let $\tilde{A}$ be the functional algebra of all finite-dimensional functions from $X_{I}$ into $\bar{B}$. Since $\bar{B}$ preserves the suprema of $B$ (i.e., if a subset of $B$ has a supremum in $B$, then it has the same supremum in $\bar{B}$ ), it follows that $A$ is a nonhomogeneous polyadic subalgebra of $\tilde{A}$. By (2.2), $\tilde{A}$ is rich. This completes the proof of the theorem.

3. The homogeneous transform of a finite-sorted nonhomogeneous algebra. The purpose of this section is to show that, to a great extent, the theory of finite-sorted nonhomogeneous algebras can be reduced to that of homogeneous algebras. We introduce first a definition. If $M$ is a nonempty set, then an $M$-sorted homogeneous polyadic algebra if a quintuple $(A, I, F, S, \exists)$ where $(A, I, S, \exists)$ is a homogeneous polyadic algebra and $F$ is a mapping from $M$ to 1-place predicates of $A$ such that

$$
\begin{aligned}
\exists(i) F_{\alpha}(i) & =1, \\
F_{\alpha}(i) \wedge F_{\beta}(i) & =0,
\end{aligned}
$$

whenever $i$ belongs to $I$ and $\alpha, \beta$ are distinct elements of $M$, and

$$
\vee\left\{F_{\alpha}(i): \alpha \in M\right\}=1 \quad \text { for all variables } i \text {. }
$$

Condition (3.3) implies that the supremum indicated exists and moreover is equal to 1 . For the remainder of this section, we assume that $M$ is a fixed nonempty finite set. It is now our purpose to introduce a technique which allows to reduce the theory of $M$-sorted nonhomogeneous algebras to the theory of $M$-sorted homogeneous algebras. To be explicit, let $(A, I, U, S, \exists)$ be an $M$-sorted locally finite nonhomogeneous algebra of infinite degree. A $U$-predicate of $A$ is a mapping $P$ from $I^{I}$ into $A$ so that $S(\tau) P(\sigma)=P(\tau \sigma)$ for all transformations $\sigma$ on $I$ and all $U$-transformations $\tau$ on $I$. Let $\tilde{A}$ be the set of all finite-dimensional $U$-predicates of $A ; \tilde{A}$ is a Boolean algebra under point-wise operations. We shall make $\tilde{A}$ into an $M$-sorted homogeneous algebra $(\tilde{A}, I, F, \tilde{S}, \tilde{\exists})$. The mapping $\tilde{S}$ is the functional 
transformation mapping. More explicitly, if $\tau$ is a transformation on $I$, then $(\tilde{S}(\tau) P)(\sigma)=P\left(\tau_{*} \sigma\right)$ for all $P$ in $\tilde{A}$ and all transformations $\sigma$ on $I$. It is easy to check that $\tilde{S}(\tau)$ maps $\tilde{A}$ into $\tilde{A}$. The operator $\tilde{\exists}$ is defined as follows. Let $i$ be a variable, $P$ an element of $\tilde{A}, J$ a finite support for $P$ and $\sigma$ a transformation on $I$. For each sort $\alpha$, let $i_{\alpha}$ be a variable in $U_{\alpha}$ so that $i_{\alpha}$ is not in $\sigma(J)$, and let $\sigma_{\alpha}$ be a transformation on $I$ so that $\sigma_{\alpha} \equiv \sigma \bmod i$ and $\sigma_{\alpha}(i)=i_{\alpha}$. Now let $(\tilde{\exists}(i) P)(\sigma)$ $=\mathrm{V}\left\{\exists\left(i_{\alpha}\right) P\left(\sigma_{\alpha}\right): \alpha \in M\right\}$.

It is a straightforward matter to check that this definition is unambiguous (i.e., independent of the choice of the support $J$ and of the variables $i_{\alpha}$ ) and that $\tilde{\exists}(i)$ is a mapping from $\tilde{A}$ into $\tilde{A}$. Using (2.1), it is also easy to see that $\tilde{\exists}(i)$ is actually the $i$-cylindrification. It follows immediately, that for every subset $J$ of $I$, the $J$-cylindrification $\tilde{\Xi}(J)$ exists on $\tilde{A}$. Therefore $(\tilde{A}, I, \tilde{S}, \tilde{\exists})$ is a functional locally finite polyadic algebra ( $A$-valued and over $I$ ) and hence a homogeneous polyadic algebra. In order to make $\tilde{A}$ into an $M$-sorted homogeneous algebra, the mapping $F$ still needs to be defined. This is done as follows: for each $i$ in $I$ and each $\alpha$ in $M$, let $F_{\alpha}(i)(\tau)=1$ when $\tau i$ is of the sort $\alpha$ and $F_{\alpha}(i)(\tau)=0$ otherwise. Then $(\tilde{A}, I, F, \tilde{S}, \tilde{\exists})$ becomes an $M$-sorted homogeneous algebra; we shall refer to it as the homogeneous transform of $A$ or simply the transform of $A$. There is a natural mapping $f$ from $\tilde{A}$ onto $A$ defined by $f P=P(\delta)$ where $\delta$ is the identity on $I$. Clearly, $f$ is a homomorphism. To show that $f$ is onto, let $p$ in $A$ and let $J$ be a finite support for $p$. Define a $U$-predicate $P$ of $A$ as follows: if $\tau$ is a transformation on $I$ for which there exists a $U$-transformation $\sigma$ so that $\tau \equiv \sigma \bmod (I-J)$, let $P(\tau)=S(\sigma) p$; otherwise, let $P(\tau)=0$. The definition of $P$ is unambiguous and moreover $P(\delta)=p$. This shows that $f$ is onto. The following lemma establishes the main properties of the mapping $f$.

(3.5) LEMMA. If $\tau$ is a U-transformation on $I$ and if $i$ is a variable of the sort $\alpha$, then $f \tilde{S}(\tau) P=S(\tau) f P$ and $f \tilde{\exists}(i)\left(P \wedge F_{\alpha}(i)\right)=\exists(i) f P$ for every $P$ in $\tilde{A}$.

Proof. The first equality is an immediate consequence of the fact that $\tau_{*} \delta=\tau$. The second equality becomes a straightforward verification if one uses the fact that $\tilde{\exists}(i)$ is the $i$-cylindrification.

The mapping $f$ will be referred to as the natural relativization of $\tilde{A}$ onto $A$. The next step is now to seek an abstract characterization of the kernel of $f$. In order to be able to formulate this characterization, we first define a mapping $\bar{F}$ from finite subsets $J$ of $I$ to elements $\bar{F}(J)$ of $\tilde{A}$ as follows. If $j=\phi$, let $\bar{F}(\phi)=1$; if $J$ is not empty let $i_{1}, \cdots, i_{n}$ be the distinct variables in $J$ and assume that they are of 
the sorts $\alpha_{1}, \cdots, \alpha_{n}$ respectively and let $\bar{F}(J)=F_{\alpha_{1}}\left(i_{1}\right) \wedge, \cdots$, $\wedge F_{\alpha_{n}}\left(i_{n}\right)$. Observe that $J$ supports $\bar{F}(J)$ and that $\bar{S}(\tau) \bar{F}(J)=\bar{F}(\tau(J))$ whenever $\tau$ is a $U$-transformation on $I$. The proof of the following lemma is a straightforward verification.

(3.6) Lemma. For any $P$ in $\tilde{A}, f P=0$ if and only if $P \wedge \bar{F}(J)=0$ whenever $J$ is a finite support for $P$.

We are now in a position to formulate and prove the main result of this section.

(3.7) Theorem. Let $A_{1}$ and $A_{2}$ be locally finite $M$-sorted nonhomogeneous algebras of infinite degree where $M$ is finite, and let $\tilde{A}_{1}$ and $\widetilde{A}_{2}$ be their homogeneous transform respectively. Then $A_{1}$ and $A_{2}$ are isomorphic if and only if $\tilde{A}_{1}$ and $\tilde{A}_{2}$ are isomorphic.

Proof. By an isomorphism between $\tilde{A}_{1}$ and $\tilde{A}_{2}$ we understand here a polyadic isomorphism that preserves the predicate mapping $F$. If $A_{1}$ and $A_{2}$ are isomorphic, then clearly $\tilde{A}_{1}$ and $\tilde{A}_{2}$ are isomorphic; this follows immediately from the definition of the homogeneous transform. Conversely, suppose $\tilde{A}_{1}$ and $\tilde{A}_{2}$ are isomorphic and let $\tilde{g}$ be an isomorphism of $\tilde{A}_{1}$ onto $\tilde{A}_{2}$. Let $f_{1}$ and $f_{2}$ be the natural relativizations of $\tilde{A}_{1}$ and $\tilde{A}_{2}$ onto $A_{1}$ and $A_{2}$ respectively. Define a mapping $g$ from $A_{1}$ onto $A_{2}$ by $g f_{1} p=f_{2} \tilde{g} p$. It follows from (3.5) and (3.6) that $g$ is unambiguously defined and that $g$ is an isomorphism from $A_{1}$ onto $A_{2}$.

\section{REFERENCES}

1. P. R. Halmos, Algebraic logic. I, Monadic algebras, Compositio Math. 12 (1955), 217-249.

2. - Algebraic Logic. II, Homogeneous locally finite polyadic Boolean algebras of infinite degree, Fund. Math. 43 (1956), 255-325.

3. - Algebraic logic. III, Predicates, terms, and operations in polyadic algebras, Trans. Amer. Math. Soc. 83 (1956), 430-470.

4. - Algebraic logic. IV, Equality in polyadic algebras, Trans. Amer. Math. Soc., 86 (1957), 1-27.

5. H. M. MacNeille, Partially ordered sets, Trans. Amer. Math. Soc. 42 (1937), 4.16-460.

6. Arnold Schmidt, Ueber dedaktiven Theorien mit mehreren Sorten von Grunddingen, Math. Ann. 115 (1938), 485-506.

7. - Die Zulaessigkeit der Behandlung mehrsortiger Theorien mittels der ueblichen Praedikalenlogik, Math. Ann. 123 (1951), 187-200.

Universite DE MonTrEAL 